\title{
Thermally Driven Electrokinetic Energy Conversion with Liquid Water Microjets
}

\author{
Royce K. Lam ${ }^{\mathrm{a}, \mathrm{b}}$, Zach Gamlieli ${ }^{\mathrm{a}}$, Stephen J. Harris ${ }^{\mathrm{c}}$, Richard J. Saykally*a,b \\ ${ }^{\mathrm{a}}$ Department of Chemistry, University of California, Berkeley, California 94720 \\ ${ }^{\mathrm{b}}$ Chemical Sciences Division, Lawrence Berkeley National Laboratory, Berkeley, \\ California 94720 \\ ${ }^{c}$ Material Sciences Division, Lawrence Berkeley National Laboratory, Berkeley, \\ California 94720
}

\author{
Corresponding Author \\ *Department of Chemistry \\ D31 Hildebrand Hall \\ University of California, Berkeley, CA 94720 \\ Phone: 510-642-1047 \\ Email: saykally@berkeley.edu
}




\title{
Graphical Abstract
}

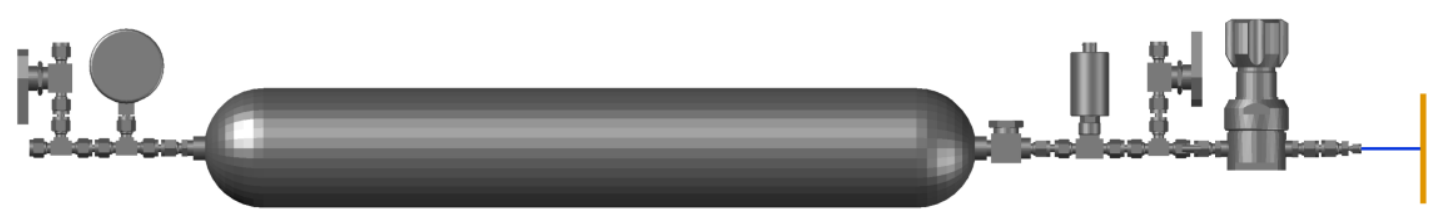

\begin{abstract}
A goal of current energy research is to design systems and devices that can efficiently exploit waste heat and utilize solar or geothermal heat energy for electrical power generation. We demonstrate a novel technique exploiting water's large coefficient of thermal expansion, wherein modest thermal gradients produce the requisite high pressure for driving fast-flowing liquid water microjets, which can effect the direct conversion of the kinetic energy into electricity and gaseous hydrogen. Waste heat in thermoelectric generating plants and combustion engines, as well as solar and geothermal energy could be used to drive these systems.
\end{abstract}

\section{Keywords}

- Renewable Energy, Waste Heat, Power Generation 


\section{Introduction}

As described in the 2014 US DOE Report "The Water-Energy Nexus: Challenges and Opportunities", 1 cooling of thermoelectric power plants accounts for $40 \%$ of US freshwater usage and dissipates tremendous quantities (27 quads/year) of primary energy as waste heat due to fundamental inefficiencies in converting thermal energy to electricity; internal combustion engines generate similarly prodigious amounts of waste heat (21.3 quads/year). Devices for mitigating this energy loss employing thermoelectric conversion schemes (i.e. solid-state thermoelectric generators and thermophotovoltaics) exist, but typically require high operating temperatures. An interesting alternative is to exploit the large coefficient of thermal expansion of liquid water to utilize waste heat via the production of high hydrostatic pressures, which can, in turn, drive recently demonstrated electrokinetic energy conversion and hydrogen production processes in fast-flowing liquid microjets..$^{2,3}$

Electrokinetic conversion of mechanical energy with liquid microjets and nanojets comprises a potentially important, but largely unexplored technology. There have been numerous studies on producing electrokinetic currents by forcing water through both porous materials or individual channels. ${ }^{4-13}$ The electrokinetic streaming current originates from the overlap between the liquid flow profile and the charge distribution near the solid-liquid interface. The streaming current $\left(I_{S}\right)$ generated by a circular channel can be described by the integral of the product of the velocity profile, $v(r)$, and the charge density distribution, $\rho(r)$

$$
I_{s}=2 \pi R \int_{0}^{R} v(r) \rho(r) d r
$$

where $\mathrm{R}$ is the channel radius and $\mathrm{r}$ is the distance from the channel wall.,2,3 Typically, these experiments generate streaming currents by moving liquid from one reservoir to another through a porous membrane or a single channel by applying pressure to a single side. The direct connection between the two reservoirs introduces significant inefficiencies in the energy conversion due to backconduction through the liquid.6,14,15 More recently, Miljkovic et al. have demonstrated that water droplets jumping between hydrophobic and hydrophilic 
surfaces can attain a positive electrostatic charge and generate electrical power via a similar charge separation mechanism. ${ }^{16,17}$

The inefficiencies resulting from back-conduction through the liquid in the reservoir systems are eliminated when liquid jets are employed. Under the laminar flow conditions of this study, the streaming current can be described by Equation $2^{18}$

$$
I_{s}=-8 \pi \varepsilon_{0} K v \zeta
$$

where $\varepsilon_{0}$ is the permittivity of free space, $K$ is the dielectric constant, $v$ is the areaaveraged flow velocity, and $\zeta$ is the zeta-potential. Preliminary studies conducted at Berkeley have demonstrated a conversion efficiency $>10 \%$ with a peak power of $\sim 6$ $\mathrm{mW}$ in water flowing from a $10 \mu \mathrm{m}$ inner diameter aperture under turbulent flow conditions ${ }^{2}$ and have shown that the electrical energy production can be accompanied by simultaneous gaseous hydrogen generation. ${ }^{3}$ Here, electrical efficiency $(\eta)$ is calculated as electrical power divided by the input kinetic energy as a function of volumetric flow rate $(Q)$ and the applied pressure differential $(\Delta P)$

$$
\eta=\frac{\mathrm{I}_{\mathrm{S}}^{2} \mathrm{R}_{\mathrm{L}}}{\mathrm{Q} \Delta \mathrm{P}}
$$

where $R_{L}$ is the load resistance. In this construct, the efficiency of the system is decoupled from the efficiency of refilling and heating the cylinder which is dependent of variety of factors (i.e. location of the water source, thermal conductivity of the reservoir, heat capacity of the reservoir, etc.). A patent has been issued for this concept and device design. ${ }^{19}$ Using a similar system to drive a droplet beam into a region of high electric field, up to $48 \%$ efficiency has been reported for in the conversion of the liquid's kinetic energy to potential energy. ${ }^{20}$

High pressures ( $>500 \mathrm{PSI}$ ) are required to force water through the microjets and generating these pressures substantially reduces the "wall plug" efficiency. Here we address this problem by demonstrating that such pressures can easily be generated by exploiting water's relatively large volumetric thermal expansion coefficient $\left(a_{v}\left(20^{\circ} \mathrm{C}\right)=2.07 \times 10^{-4} \mathrm{~K}^{-1}\right)$ and moderate bulk modulus $\left(K_{T}=3.16 \times 10^{5}\right.$ PSI). The resulting pressure change $(\Delta P)$ induced by the temperature increase $(\Delta \mathrm{T})$ can therefore be approximated by 
For liquid water in a rigid container, we can therefore expect generate $65.41 \mathrm{PSI} /{ }^{\circ} \mathrm{C}$. Below we describe the design and construction of practical devices that can produce useful amounts of electricity while simultaneously generating hydrogen gas from flowing water, using simple passive thermal sources to produce the requisite high water pressures.

\section{Experimental Design and Discussion}

Figure 1 presents a schematic of the experimental configuration employed in this exploratory study. A $3.785 \mathrm{~L}$ double-ended stainless steel sample cylinder, filled with deionized water, is used for the liquid reservoir. In the current design, the sample cylinder limits the maximum pressure to below 1450 PSI. A digital pressure transducer (Heise Model DXD) provides pressure and temperature readings prior to the jet assembly, which has been described in detail previously in our recent electrokinetic energy conversion ${ }^{2,3}$ and interfacial ion affinity studies ${ }^{21}$. Briefly, the liquid microjet is produced by ejecting the pressurized water from a $30 \mu \mathrm{m}$ inner diameter silica capillary rather than the Pt/Ir electron microscope apertures used in our previous energy conversion experiments. Silica capillaries were employed here due to their intrinsic chemical stability. However, due to the laminar flow profile and the significantly lower $\xi$ potential of silica relative to that of the metal apertures, the electrokinetic conversion efficiency was not measured for this system. The liquid beam travels $\sim 5 \mathrm{~cm}$ in ambient air at average linear flow velocities of $\sim 20 \mathrm{~m} / \mathrm{s}$ before impinging on a copper plate connected to an ammeter.

In order to maintain the necessary backing pressure, the temperature must slowly be increased as the water is expelled from the tank. The heating and liquid flow are controlled using a feedback loop which couples the heating element, the liquid flow as a function of pressure, and the tank pressure. In this scheme, the heating element is activated or deactivated when the pressure falls below 1200 PSI or rises above 1300 PSI, respectively. The back pressure regulator is set to open when the pressure rises above $\sim 1200$. The current generated is monitored within this feedback loop. Internal temperature measurements are collected using a T-type thermocouple inserted into the center of the cylinder. The external temperature is 
measured using a digital temperature probe (Thorlabs TSP01). The cylinder is heated resistively using a heating tape system coupled to a digital AC switch (CPS U-X3) and the system pressure. The generated current is measured using a current amplifier connected to the copper target electrode (FEMTO DDPCA-300). Instrument control and data acquisition are managed using Labview.

Figure 2 shows the temperature, pressure, and current measured for an individual experiment. The temperature (Figure 2a) is monitored in three different locations on the cylinder - internal (red), external (blue), and behind the silica capillary (black). During a typical experiment, the initial liquid temperature is $\sim 25$ ${ }^{\circ} \mathrm{C}$ and ultimately reaches temperatures of above $100^{\circ} \mathrm{C}$. The pressure generated from the heating is shown in Figure $2 \mathrm{~b}$. The sample cylinder is initially filled with deionized water at a backing pressure of $\sim 30-35$ PSI and is allowed to reach a maximum pressure of $\sim 1350$ PSI. The initial pressurization, defined as the time required to reach 1200 PSI, typically takes $\sim 50$ minutes and requires a temperature change of $\sim 20^{\circ} \mathrm{C}\left(\sim 60 \mathrm{PSI} /{ }^{\circ} \mathrm{C}\right)$ which is in good agreement with our estimate from Equation 4. Figure 2c shows the current generated by the system as a function of time for a single experiment. The on/off behavior of the generated current is a direct result of the opening and closing of the back pressure regulator. In our present system, wherein the reservoir is not insulated, the pressure rise resulting from the heating does not keep pace with the pressure drop resulting from the liquid microjet. This can easily be corrected in practical situations.

\section{Conclusions}

We envision using EK power stations to supply or supplement power to buildings and devices, and employing hydrogen fuel cells that use the hydrogen coproduced with the electricity. High pressures are required to drive water through the microjets, but such pressures $\left(\sim 60 \mathrm{PSI} /{ }^{\circ} \mathrm{C}\right)$ can be obtained "for free" (from waste heat sources) by taking advantage of water's large thermal expansion coefficient and heating it in a rigid (e.g. metal) chamber with solar energy or with waste heat. In this way, electrokinetic jets can work in tandem with more conventional thermoelectric devices, which typically require much higher temperatures for good efficiency. ${ }^{19}$ 


\section{References}

(1) Water Energy Nexus: Challenges and Opportunities; 2014.

(2) Duffin, A.; Saykally, R. Electrokinetic Power Generation from Liquid Water Microjets. J. Phys. Chem. C 2008, 112 (43), 17018-17022.

(3) Duffin, A.; Saykally, R. Electrokinetic Hydrogen Generation from Liquid Water Microjets. J. Phys. Chem. C 2007, 111 (32), 12031-12037.

(4) Siria, A.; Poncharal, P.; Biance, A.-L.; Fulcrand, R.; Blase, X.; Purcell, S. T.; Bocquet, L. Giant Osmotic Energy Conversion Measured in a Single Transmembrane Boron Nitride Nanotube. Nature 2013, 494 (7438), 455-458.

(5) Chang, C.-C.; Yang, R.-J. Electrokinetic Energy Conversion Efficiency in IonSelective Nanopores. Appl. Phys. Lett. 2011, 99 (8), 083102.

(6) van der Heyden, F. H. J.; Bonthuis, D. J.; Stein, D.; Meyer, C.; Dekker, C. Power Generation by Pressure-Driven Transport of Ions in Nanofluidic Channels. Nano Lett. 2007, 7 (4), 1022-1025.

(7) van der Heyden, F. H. J.; Bonthuis, D. J.; Stein, D.; Meyer, C.; Dekker, C. Electrokinetic Energy Conversion Efficiency in Nanofluidic Channels. Nano Lett. 2006, 6 (10), 2232-2237.

(8) Delgado, A. V.; González-Caballero, F.; Hunter, R. J.; Koopal, L. K.; Lyklema, J. Measurement and Interpretation of Electrokinetic Phenomena (IUPAC Technical Report). Pure Appl. Chem. 2005, 77 (10), 1753.

(9) Canning, J.; Buckley, E.; Lyytikainen, K. Electrokinetic Air-silica Structured Multi-Microchannel Capillary Batteries. Electron. Lett. 2004, 40 (5), 298.

(10) Canning, J.; Buckley, E.; Huntington, S.; Lyytikäinen, K. Using MultiMicrochannel Capillaries for Determination of the Zeta Potential of a Microfluidic Channel. Electrochim. Acta 2004, 49 (21), 3581-3586.

(11) Kwok, J. Y. and F. L. and L. W. K. and D. Y. Electrokinetic Microchannel Battery by Means of Electrokinetic and Microfluidic Phenomena. J. Micromechanics Microengineering 2003, 13 (6), 963.

(12) Werner, C.; Körber, H.; Zimmermann, R.; Dukhin, S.; Jacobasch, H. Extended Electrokinetic Characterization of Flat Solid Surfaces. J. Colloid Interface Sci. 1998, 208 (1), 329-346.

(13) Werner, C.; Zimmermann, R.; Kratzmüller, T. Streaming Potential and 
Streaming Current Measurements at Planar Solid/liquid Interfaces for Simultaneous Determination of Zeta Potential and Surface Conductivity. Colloids Surfaces A Physicochem. Eng. Asp. 2001, 192 (1-3), 205-213.

(14) Davidson, C.; Xuan, X. Effects of Stern Layer Conductance on Electrokinetic Energy Conversion in Nanofluidic Channels. Electrophoresis 2008, 29 (5), 1125-1130.

(15) Lyklema, J. Surface Conduction. J. Phys. Condens. Matter 2001, 13 (21), 5027.

(16) Miljkovic, N.; Preston, D. J.; Enright, R.; Wang, E. N. Electrostatic Charging of Jumping Droplets. Nat Commun 2013, 4.

(17) Miljkovic, N.; Preston, D. J.; Enright, R.; Wang, E. N. Jumping-Droplet Electrostatic Energy Harvesting. Appl. Phys. Lett. 2014, 105 (1), 013111.

(18) Groves, J. N.; Sears, A. R. Alternating Streaming Current Measurements. J. Colloid Interface Sci. 1975, 53 (1), 83-89.

(19) Saykally, R. J.; Duffin, A. M.; Wilson, K. R.; Rude, B. S. Method and Apparatus for Electrokinetic Co-Generation of Hydrogen and Electric Power from Liquid Water Microjets. 8372374, 2013.

(20) Xie, Y.; Bos, D.; de Vreede, L. J.; de Boer, H. L.; van der Meulen, M.-J.; Versluis, M.; Sprenkels, A. J.; van den Berg, A.; Eijkel, J. C. T. High-Efficiency Ballistic Electrostatic Generator Using Microdroplets. Nat Commun 2014, 5.

(21) Kelly, D. N.; Lam, R. K.; Duffin, A. M.; Saykally, R. J. Exploring Solid/Aqueous Interfaces with Ultradilute Electrokinetic Analysis of Liquid Microjets. J. Phys. Chem. C 2013, 117 (24), 12702-12706.

\section{Acknowledgments}

This work was supported by the Director, Office of Basic Energy Sciences, Office of Science, U.S. Department of Energy (DOE) under Contract No. DE-AC02-05CH11231, through LBNL Chemical Sciences Division. The data presented are available upon request to saykally@berkeley.edu. 


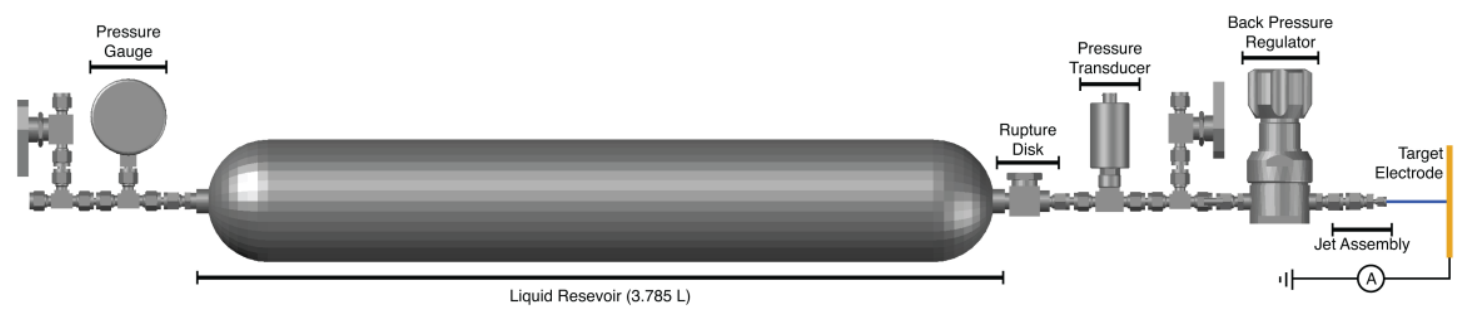

Figure 1 Schematic of the thermal electrokinetic microjet apparatus. The streaming current is collected at a copper target electrode placed $\sim 5 \mathrm{~cm}$ away from the silica capillary using a current amplifier. 


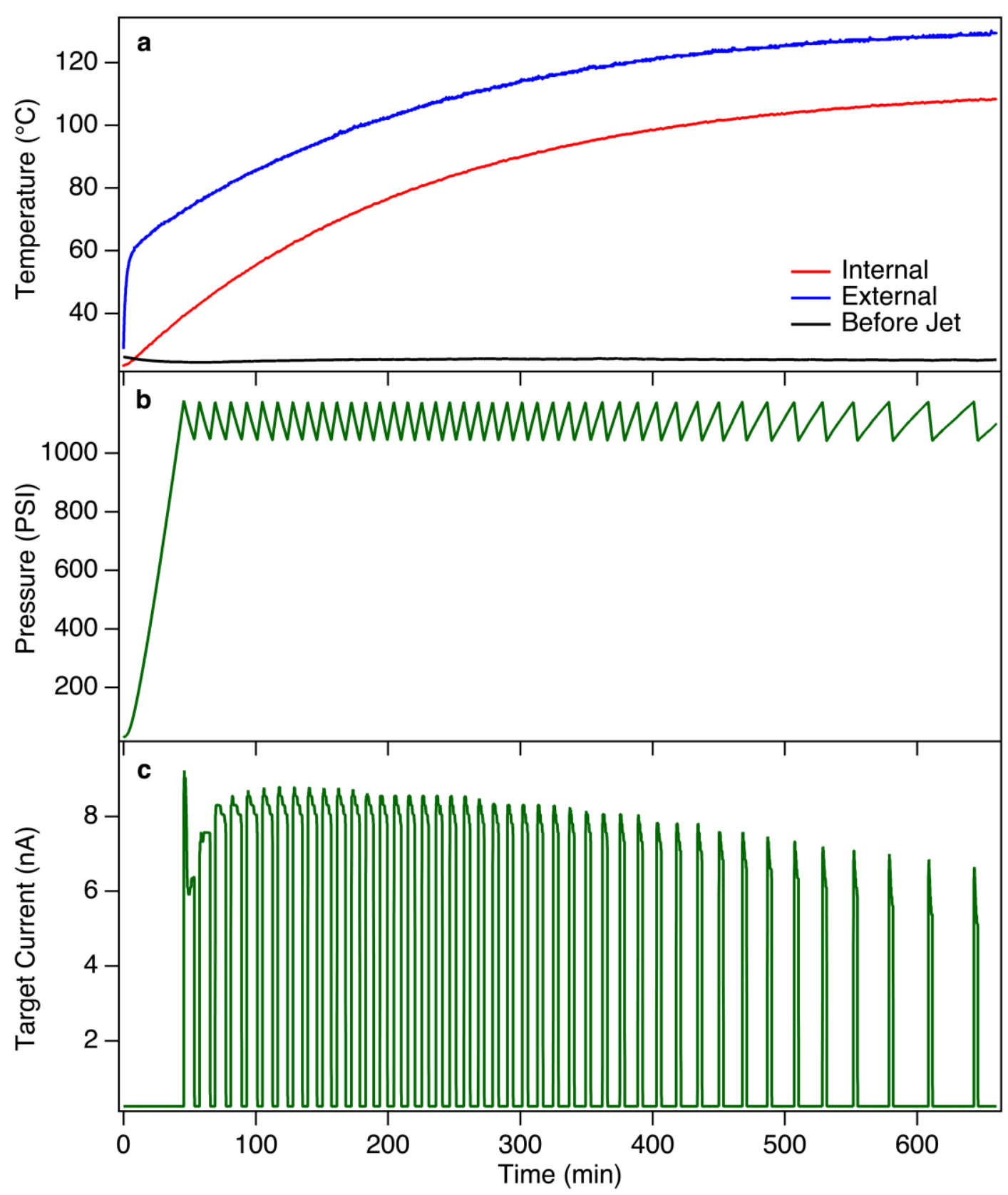

Figure 2 Typical data from a single experiment. (a) Internal (red), external (blue), and before jet (black) temperature vs time. (b) Pressure vs time. (c) Target current vs time. All measurements were collected simultaneously with a time step of $1 \mathrm{~s}$. 